\title{
Exploring science and technology through the Herschel space observatory
}

\author{
V. Minier ${ }^{1}$ and M. Rouzé ${ }^{2}$ \\ ${ }^{1}$ CEA/Irfu, Service d'astrophysique, 91191 Gif-sur-Yvette, France \\ email: vincent.minier@cea.fr \\ ${ }^{2} \mathrm{CNES}, \mathrm{CDT} / \mathrm{ME} / \mathrm{EU}, 18$ av. Edouard Belin, 31401 Toulouse, France
}

\begin{abstract}
Because modern astronomy associates the quest of our origins and high-tech instruments, communicating and teaching astronomy explore both science and technology. We report here on our work in communicating astronomy to the public through Web sites (www.herschel.fr), movies on Dailymotion (www.dailymotion.com/AstrophysiqueTV) and new ITC tools that describe interactively the technological dimension of a space mission for astrophysics.
\end{abstract}

Keywords. communication, history and philosophy of astronomy, techniques: photometric

We report on a project aiming to communicate Herschel space observatory results to the French-speaking public. This public outreach effort is complementary to the European Space Agency (ESA) communication on Herschel. The project started in 2008 at CEA in France and then became national (CNES - CEA - CNRS) in 2009 following the successful launch of Herschel on May, 14th 2009. The project is mainly based on Web resources with the creation of a dedicated Web site: www.herschel.fr. The Web site includes 3 levels of reading: a home page with videos and news board; sections dedicated to science topics and instrumentation; applications, videos and texts to go further in detail in the Herschel mission. An important effort has been made to produce videos of high quality with professional motion designers. Many videos include interviews of scientists presenting their goals, and historical background given by decision-makers and mission principal investigators. Other videos propose 3D animation of the Herschel observations (Picturing Star Motion) using colour-coding and effects in precise agreement with scientific data (intensity, distance, depth, coordinates). These videos are available in the Multimedia area as well as in streaming on the Astrophysique TV Dailymotion channel. These resources provide background for science journalists, educators and general public, and have been used outside the Internet, in exhibitions, teaching, public talks, movie theatres... Based on statistics, we estimate that more than 200,000 people have been reached via Internet. The secondary impact is difficult to estimate. All the archives used for this project feed an historical study of Herschel invention led by V. Minier.

\section{Acknowledgements}

We would like to acknowledge the work in this project of Thierry Morin (CEA Irfu) and Lucille Colombel (CEA DCom) on the Web design and development, Pierre-Francois Didek (Karamoja Productions) on the Web documentaries, Paco Abelleira and Laurent Fouilloux (Novae Factory) on 3D animations and interactive application, the support of Philippe Chauvin (CNRS/INSU) and all the astronomers who contributed directly to the production of contents or indirectly through their scientific results. This project has been funded mainly by CNES, the French Space Agency, and CEA. Web applications summarising the studies in history of techniques conducted at CEA Larsim were partly supported by the Campus Spatial - Université Paris Diderot (project Astro Innov). 